\title{
Quiste de conducto de Skene en niñas. A propósito de 2 casos clínicos
}

\author{
A. Miño • J. Rodríguez
}

\begin{abstract}
\section{Skene duct cyst in female newborns - Case reports}

Introduction: The Skene duct cyst, classified as a paraurethral cyst, is a rare congenital abnormality in female neonates and it may manifest throughout the course of life. The incidence varies from 1 in 2000 to 3000 female births. Objective: To expose the characteristics and symptoms of a Skene duct cyst at different stages of child development in order to carry out a timely suspicion and diagnosis. Case report: The first case is a female newborn who presented a painless yellowish tumor adjacent to the urethral meatus, which drained spontaneously; the second case is a teenager who consulted due to leucorrhea for a year and a three-centimeter diameter paraurethral injury between the labia minora, which required surgical treatment and study of the paraurethral mass. Conclusions: Based on the literature review, we concluded that the frequency of Skene duct cyst is higher than the number of reported cases. Cyst removal, marsupialization, puncture and aspiration are all effective treatment methods. Spontaneous drainage is also appropriate in certain cases. We suggest the use of the least aggressive technique according to each case.
\end{abstract}

(Key words: Cyst of Skene, Paraurethral cyst, Skene duct).

Rev Chil Pediatr 2014; 85 (5): 584-587

\section{RESUMEN}

Introducción: El quiste del conducto de Skene, se describe dentro de los quistes parauretrales, siendo una rara anomalía congénita descrita en neonatos de sexo femenino, que se puede presentar en el transcurso de la vida. La incidencia varía de 1 cada 2.000-3.000 recién nacidos vivos femeninos. Objetivo: Dar a conocer las características y formas de presentación del quiste del conducto de Skene en distintas etapas del desarrollo del niño para su sospecha y diagnóstico oportuno. Casos clínicos: Se presenta una recién nacida con una lesión tumoral amarillenta, adyacente al meato uretral, no dolorosa, que drena espontáneamente y un segundo caso de una adolescente que consulto por leucorrea de un año de evolución, con una lesión parauretral de tres centímetros de diámetro entre labios menores, que requirió tratamiento quirúrgico y estudio de la masa parauretral. Conclusiones: Basado en la revisión de la literatura, concluimos que la frecuencia de los quistes de conducto de Skene es más alta que el número de casos informados. La extirpación, marsupialización, la punción y aspiración del quiste son todos métodos eficaces de tratamiento. El drenaje espontáneo también es una conducta apropiada en ciertos casos. Se sugiere utilizar la técnica menos agresiva acorde a cada caso.

(Palabras clave: Quiste de Skene, Quiste Parauretral, Conducto de Skene).

Rev Chil Pediatr 2014; 85 (5): 584-587

Recibido el 08 de mayo de 2014, última versión aceptada el 03 de octubre de 2014.

Alberto Miño Barrera ( $\square)$

Médico Cirujano. Hospital San Juan de Dios de Curicó.

E-mail: albertomag74@hotmail.com

Jorge Rodríguez Herrera

Urólogo Pediátrico. Hospital Luis Calvo Mackenna. 


\section{Introducción}

El quiste del conducto de Skene, es una rara anomalía congénita descrita en neonatos de sexo femenino, pudiendo estar presente en el transcurso de su desarrollo. Se trata de un aumento de volumen ovoideo, amarillento o anaranjado, no dolorosa, con vascularización superficial, que se ubica adyacente al meato uretral. Su incidencia varia según los reportes, estimándose tasas cercanas a 1 cada 2.0003.000 recién nacidos vivos femeninos ${ }^{1}$, pero es probable que esta incidencia esté subestimada, dado que muchos casos pueden no ser reportados dada su benignidad ${ }^{2,3}$.

La lesión depende de las glándulas parauretrales femeninas, que son homologas a la próstata masculina ${ }^{4,5}$. Son pequeñas glándulas en número de 6 a $30^{2-6}$ cuyo conducto excretor desemboca en el tercio distal de la uretra o en el introito vaginal. Existen dos de mayor tamaño que reciben el nombre de glándulas de Skene, su función es lubricar el introito mediante secreción mucoide durante la estimulación sexual.

Esta patología se debe incluir en el diagnóstico diferencial con otras masas interlabiales, como el Quiste de Gärtner, quiste de inclusión epitelial, uretrocele ectópico, himen imperforado y el sarcoma botroide ${ }^{1-4,7}$.

\section{Objetivo}

Dar a conocer las características y formas de presentación del quiste del conducto de Skene en distintas etapas del desarrollo del niño, para su sospecha y diagnóstico oportuno.

\section{Caso clínico 1}

Recién nacida hija de madre primigesta de 37 años, con embarazo controlado de 38 semanas. Se practicó cesárea de urgencia por restricción del crecimiento intrauterino, obteniendo un recién nacido pequeño para la edad gestacional. Al examen destacaban genitales femeninos con tumoración redonda amarillenta o anaranjada con vasos en la superficie, adyacente al meato uretral; con diuresis espontánea.

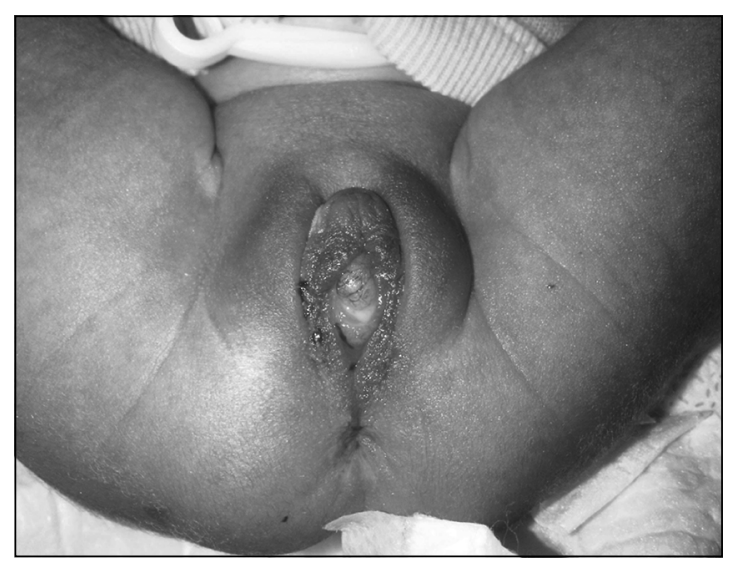

Figura 1. Se observa Quiste de Skene, con salida de contenido lechoso en recién nacido.

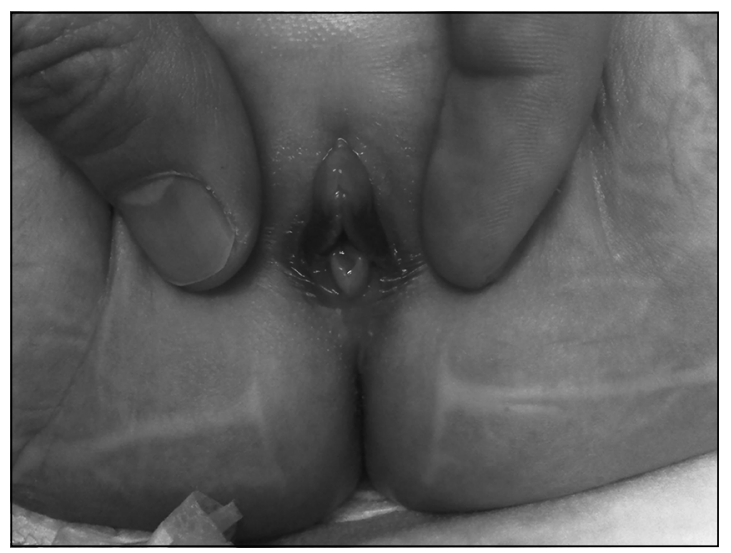

Figura 2. Se observa resolución espontánea de Quiste de Skene.

Evaluada por cirujano infantil al día de vida, apreciando quiste que respetaba el meato urinario, no doloroso a la palpación y que se drenó parcialmente a la movilización de éste en el examen, dando salida a líquido lechoso. Ante los hallazgos se diagnosticó un Quiste de Skene.

Se controló a los 2 meses de edad apreciando la resolución completa del quiste, sin requerir tratamiento quirúrgico (figuras 1 y 2).

\section{Caso clínico 2}

Adolescente de 14 años, derivada a ginecología por cuadro de leucorrea de un año de 


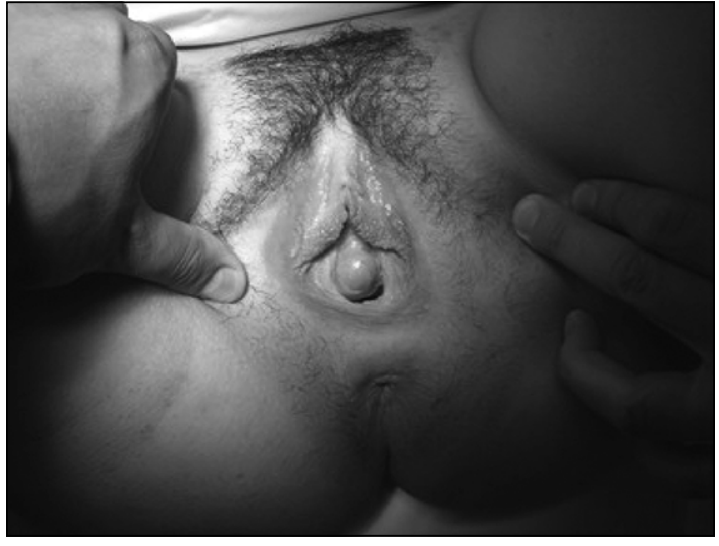

Figura 3. Quiste Skene en adolescente.

evolución. La paciente habría iniciado su menarquia a los 11 años, con ciclos menstruales regulares, dismenorrea no invalidante y caracteres sexuales secundarios normales. En la evaluación ginecológica destacó quiste de 3 $\mathrm{cm}$ de diámetro entre labios menores, tenso, no doloroso, parauretral derecho y no se observaba meato uretral. Se evaluó por urología y se realizó estudio con Ecografía renal y vesical, en la que destacó un quiste vulvar de contenido espeso de $1,7 \times 0,6 \mathrm{~cm}$.

Ante los hallazgos se realizó cistogenitoscopía, extirpación de quiste y biopsia, procedimiento realizado sin incidentes. Con la biopsia se confirmó el diagnóstico de Quiste de Skene (figuras 3, 4 y 5).

\section{Discusión}

Las glándulas de Skene fueron descubiertas y descritas por Alexander Johnston Chalmers Skene, en $1880^{6}$. El conducto de Skene es uno de casi 30 conductos que se abren dentro o alrededor del meato uretral femenino. La etiología exacta es desconocida, pero la obstrucción de los conductos de Skene a consecuencia de la infección o inflamación ha sido postulada. Los quistes parauretrales son poco comunes en cualquier categoría de edad, pero en particular en recién nacidos. La mayor parte de los quistes parauretrales son unilaterales y predominantemente derecho, aunque se han descrito bilaterales ${ }^{5}$.

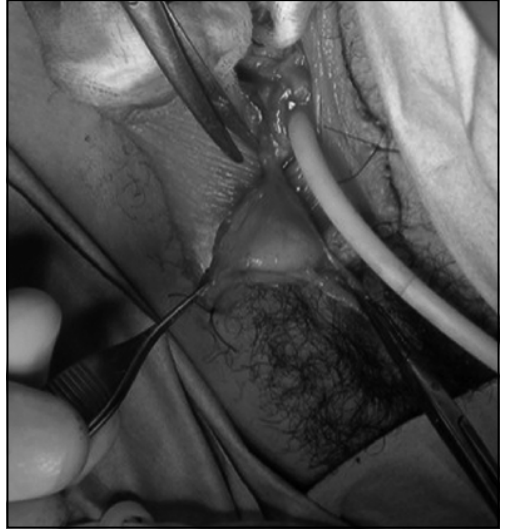

Figura 4. Extirpación quirúrgica de quiste, cateterizando previamente uretra.

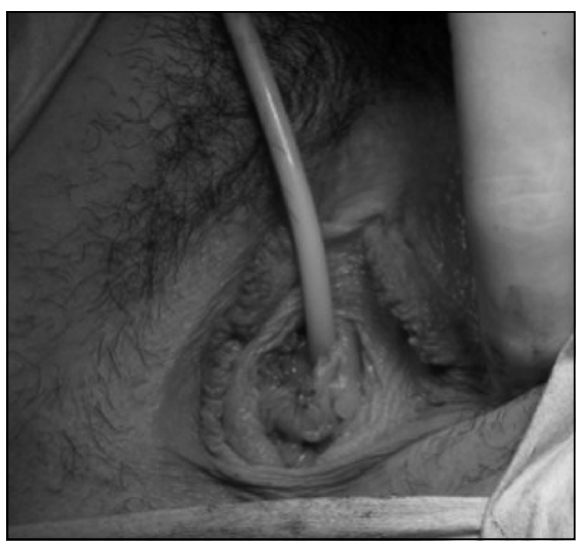

Figura 5. Región genital post extirpación de quiste de Skene.

La causa de obstrucción ductal en recién nacidos es desconocida ${ }^{4,5}$. Wrigth postuló que las hormonas femeninas adquirida maternalmente pueden provocar secreción glandular y causar la formación de quistes en neonatos ${ }^{2,5,8}$. Informes recientes destacan que los quistes en general se solucionan espontáneamente después de tres meses de nacido, debido a la baja de estas hormonas femeninas ${ }^{2}$. Rara vez los quistes parauretrales se presentan con dolor o descarga vaginal, síntomas descritos con mayor frecuencia en niñas mayores y adolescentes.

La verdadera incidencia de los quistes de Skene no esta clara por lo descrito en la literatura de distintos países ${ }^{6}$. Merlob relató 6 casos 
entre 3.026 neonatos femeninos y Lee \& Kim sólo un caso entre 2.074 niñas recién nacidas. Lo que hace pensar que la incidencia de los quistes de Skene es más alta que las cifras publicadas.

Según lo informado por Fathi y Pinter sus casos de quistes de conducto de Skene tenían los hallazgos clínicos y morfológicos descritos por Lee \& Kim. Se realizó la incisión y destechamiento de los quistes, respondiendo adecuadamente y sin recurrencia.

La aspiración con aguja parece ser conveniente y segura, sobre todo en niñas sintomáticas, además puede ayudar a aliviar la ansiedad de los padres ${ }^{6,8}$. El tratamiento conservador es también apropiado en muchas circunstancias, en especial en lesiones asintomáticas ${ }^{2,5,6}$. En los caso de obstrucción urinaria u otra complicación se ha planteado el tratamiento quirúrgico como marsupialización y/o extirpación ${ }^{2}$, esta técnica también está indicada en casos de adolescentes y adultos.

El diagnóstico es fácilmente hecho por el examen físico, sin embargo, podría necesitar de una evaluación urológica completa para realizar un diagnóstico diferencial con el quiste de conducto de Skene ${ }^{5}$. El diagnóstico definitivo esta basado en la apariencia histológica del revestimiento de la pared del quiste, el cual podría ser epitelio transitorio o epitelio escamoso $^{4}$.

\section{Conclusión}

El diagnóstico de Quiste de Skene se realiza mediante el examen físico, sin embargo, puede ser necesario estudios complementarios con ecografía para un diagnóstico diferencial con las otras masas parauretrales en los casos dudosos como en las presentaciones tardías de estos quistes.

Casos de recién nacidos que presentan este quiste son relativamente poco común en la literatura, siendo en realidad mayor su incidencia por la ausencia de diagnóstico en algunos neonatos, por su benignidad y forma asintomática.

Se plantea la conducta expectante como tratamiento de primera línea, en el que se encuentra el drenaje espontáneo y en el caso de quistes sintomáticos de recién nacidos el procedimiento por punción directa se puede considerar como el más aceptado.

En el caso de niñas mayores el tratamiento primordial sería el quirúrgico, como la extirpación y marsupialización, donde el tratamiento por punción no ha demostrado ser más efectivo y en el cual se han apreciado algunas complicaciones como infecciones debido a este procedimiento.

Potenciales conflictos de interés: Este trabajo cumple con los requisitos sobre consentimiento/asentimiento informado, comité de ética, financiamiento, estudios animales y sobre la ausencia de conflictos de intereses según corresponda.

\section{Referencias}

1.- Parente A, Angulo J, Del Cañizo A, Sánchez O, Romero $R$, Vázquez J: Quiste parauretral de Skene en niña recién nacida. Actas Urol Esp 2006; 30: 93-4.

2.- Yilmaz Y, Celik I, Dizdar E, et al: Paraurethral cyst in two female newborns: Which therapy option? Scand J Urol Nephrol 2012; 46: 78-80.

3.- Avello I, Portela O, Carrasco D, Ortega M, Ortiz A, Domínguez A: Quiste del conducto de Skene. Presentación de un caso. Mediciego 2011; 17: supl 1.

4.- Lee N, Kim S: Skene's Duct Cysts in Female Newborns. J Pediatr Surg 1992; 27: 15-7.

5.- Soyer T, Aydemir E, Atmaca E: Paraurethral Cysts in Female Newborns: Role of Maternal Estrogens. J Pediatr Adolesc Gynecol 2007; 20: 249-51.

6.- Fathi K, Pinter A: Paraurethral cysts in female neonates. Case reports. Acta Paediatr 2003; 92: 758-9.

7.- Durakbasa C, Okur H: Paraurethral Skene's Duct Cyst in a Newborn. Indian Pediatrics 2010; 47: 182.

8.- Johnson C, Millard S, Wang M, Ehsanipoor R: Prenatal diagnosis of a paraurethral cyst. Journal of Pediatric Urology 2013; 9: 91-3. 